\title{
Atomistic insights on the influence of pre- oxide shell layer and size on the compressive mechanical properties of nickel nanowires
}

Cite as: J. Appl. Phys. 125, 165102 (2019); https://doi.org/10.1063/1.5080640

Submitted: 10 November 2018 . Accepted: 13 March 2019 . Published Online: 23 April 2019

Gurcan Aral (D), Md Mahbubul Islam (D), Yun-Jiang Wang (D), Shigenobu Ogata (D), and Adri C. T. van Duin (i)
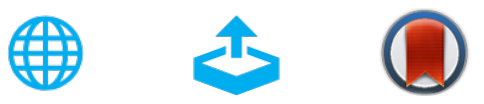

View Online

Export Citation

\section{ARTICLES YOU MAY BE INTERESTED IN}

Incorporating a soft ordered phase into an amorphous configuration enhances its uniform plastic deformation under shear

AIP Advances 9, 015329 (2019); https://doi.org/10.1063/1.5064499

Spatial modulation of heat source for highly sensitive photothermal detection

Applied Physics Letters 114, 131902 (2019); https://doi.org/10.1063/1.5080163

Applied Physics Reviews Now accepting original research

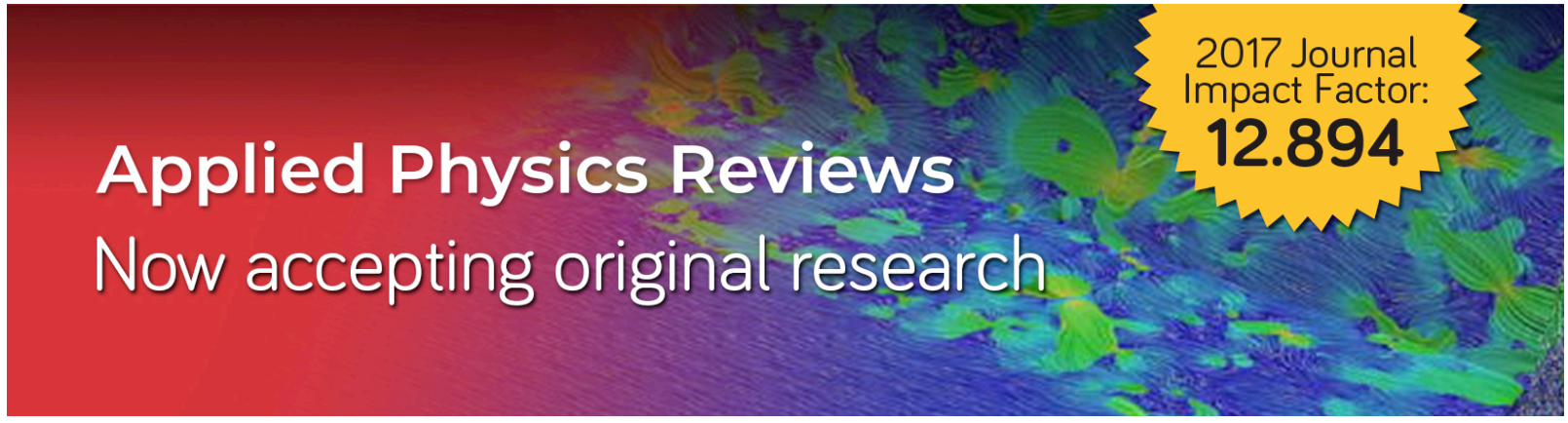




\title{
Atomistic insights on the influence of pre-oxide shell layer and size on the compressive mechanical properties of nickel nanowires
}

\author{
Cite as: J. Appl. Phys. 125, 165102 (2019); doi: 10.1063/1.5080640 \\ Submitted: 10 November 2018 - Accepted: 13 March 2019 . \\ Published Online: 23 April 2019
}

Gurcan Aral, $^{7, a)}$ (D) Md Mahbubul Islam, ${ }^{2}$ (D) Yun-Jiang Wang, ${ }^{3}$ (D) Shigenobu Ogata, ${ }^{4,5}$ (D) and Adri C. T. van Duin ${ }^{6}$ (D)

\author{
AFFILIATIONS \\ ${ }^{1}$ Department of Physics, Izmir Institute of Technology, Urla, Izmir 35430, Turkey \\ ${ }^{2}$ School of Materials Engineering, Purdue University, West Lafayette, Indiana 47907, USA \\ ${ }^{3}$ State Key Laboratory of Nonlinear Mechanics, Institute of Mechanics, Chinese Academy of Sciences, Beijing 100190, China \\ ${ }^{4}$ Department of Mechanical Science and Bioengineering, Osaka University, Osaka 560-8531, Japan \\ ${ }^{5}$ Center for Elements Strategy Initiative for Structural Materials (ESISM), Kyoto University, Kyoto 606-8501, Japan \\ ${ }^{6}$ Department of Mechanical and Nuclear Engineering, Pennsylvania State University, University Park, State College, \\ Pennsylvania 16801, USA
}

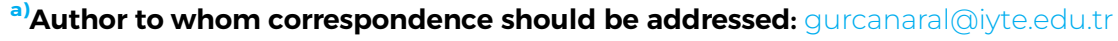

\begin{abstract}
We used ReaxFF reactive molecular dynamics simulations to systematically investigate the effects of a pre-oxide shell layer on the mechanical properties of [001]-oriented nickel $(\mathrm{Ni})$ nanowires $(\mathrm{NWs})$ under the uniaxial compressive loading at room temperature. The pristine Ni NWs are considered as references to compare the mechanical properties of the oxide-coated NWs. We found that the mechanical properties of pristine Ni NWs under uniaxial compression are sensitive to both the diameter of the NWs and the pre-oxide shell layer, and their combined effect determines the overall stress and strain behaviors. The compressive strength of the pristine NWs decreases significantly with the decreasing diameter. We observe that the native defected amorphous pre-oxide shell layer with $\sim 1.0 \mathrm{~nm}$ thickness leads to a lowering of the mechanical compressive resistivity of NWs and causes additional softening. Oxide-coated NWs exhibit a lesser size-dependent unique properties and a lower overall yield strength compared to their pristine counterparts. The reduction of the mechanical compressive yield stress and strain with the decreasing diameter is due to the substantial changes in plastic flow as well as correlated with the existence of the preoxide shell layer as compared to its pristine counterpart. Particularly, pre-oxide shell layers have pronounced effects on the initiation of initial dislocations to onset plastic deformation and consequently on the overall plastic response.
\end{abstract}

Published under license by AIP Publishing. https://doi.org/10.1063/1.5080640

\section{INTRODUCTION}

The use of metallic nanowires (NWs), such as Ni NWs, is ubiquitously subjected to reactive oxidizing environments, which leads to the formation of a pre-oxide shell layer on the free surface. $^{1-8}$ This thin pre-oxidized shell layer on the free surface of $\mathrm{Ni}$ NWs forms the core-shell (crystalline Ni-amorphous $\mathrm{Ni}_{\mathrm{x}} \mathrm{O}_{\mathrm{y}}$ ) nanostructures that alter the local microstructure, create interfaces between the core and the shell layer, and introduce a large number of surface structural defects, which are significantly distinguishable from their pristine counterparts. ${ }^{2-5,7,9}$ These effects of the coreshell structure lead to changes in the physical, mechanical, and chemical characteristics of the pristine Ni NWs. ${ }^{1,9-11}$ At the nanoscale, these features are quite significant due to the high surfaceto-volume ratios of NWs and thus warrants special attention in both experimental and theoretical studies. ${ }^{1,3,5,12,13}$ In particular, a comprehensive understanding of the underlying atomistic mechanisms of oxidation of reactive nanomaterials, their effects on the microstructure, and materials' properties are of evident necessity. ${ }^{4,8,9}$ Interestingly, such existence of an inherent native pre-oxide shell layer results in the emergence of unique physical, ${ }^{2}$ optical, mechanical, ${ }^{2,10}$ and chemical properties. ${ }^{14}$ Oftentimes, this feature renders surface oxide coating of Ni NWs of beneficial practical 
interest, and its technological importance has gained increased significance in a variety of nanoscience applications. ${ }^{1}$ The change in surface chemical composition, stoichiometry, morphology, microstructure, defects, and thickness of oxide shell layers dictate crucially the overall functionalities of nanomaterials. ${ }^{9,10,15}$ The modification of NWs through the formation of an oxide layer opens up opportunities for functionalization of NWs with novel structures, thus controlling the related properties for potential nanoengineering applications. ${ }^{1,9,15}$ For example, the functionalization of the magnetic performance and related properties of the oxide-coated Ni NWs is associated with the size, length, and morphology compared to the unoxidized counterparts of the corresponding size., ${ }^{1,15}$

The accurate prediction of the effects of surface pre-oxide layers on the mechanical deformation properties of the pristine $\mathrm{Ni}$ NWs is practically essential to ensure the mechanical stability, reproducibility, and reliability of NWs. ${ }^{10,14}$ The required spatiotemporal resolutions to investigate the oxidation and the effect of oxide surface are still prohibitive for state-of-the-art experimental techniques. ${ }^{12,13}$ Therefore, theoretical predictions can play a pivotal role in designing the nanomaterials. Since reactive nanomaterials readily react to form an oxide layer, for a direct comparison with experiments it is inevitable to consider the pre-oxide shell layer on metallic NWs into the theoretical calculation. However, the nature of oxide-coated metallic NWs, including defects, and the coexistence of various mixed interatomic bonding make their surface oxide effects on the mechanical properties highly difficult to simulate by using fixed charge interatomic potentials. ${ }^{7}$ However, the sizedependent mechanical deformation properties and the mechanism of single crystalline Ni NWs have been widely reported for similar experimental conditions and parameters such as different crystalline orientations, loading conditions, strain rate, loading direction, and temperature. ${ }^{16-19}$ The capability of the environment dependent variable charge scheme of the ReaxFF force field method rendered it possible to examine a variety of chemomechanical deformation processes associated with the pre-oxidized shell layer using the classical molecular dynamics (MD) simulations. The reactive potentials are capable of simulating reactions and characterization of the nature of oxidation through the fly bond breaking and formation during MD simulations. The ReaxFF method is also capable of describing the atomic interaction including the coexistence of metallic, ionic, and covalent interatomic bonding such as $\mathrm{Ni} / \mathrm{O} / \mathrm{H}$ systems. ${ }^{7,20}$ This method has been established to accurately reproduce experimental data for a wide range of physical and chemical properties of $\mathrm{Ni} / \mathrm{O} / \mathrm{H}$ related systems. ${ }^{20}$ A number of computational studies with ReaxFF have been used "to identify the role of" the pre-oxidation shell layer coupled with the mechanical deformation properties for the coreshell NW systems, such as core-shell structures of $\mathrm{Al} / \mathrm{Al}_{\mathrm{x}} \mathrm{O}_{\mathrm{y}}{ }^{21,22}$ $\mathrm{Ni} / \mathrm{Ni}_{\mathrm{x}} \mathrm{O}_{\mathrm{y}}{ }^{2}$, and $\mathrm{Fe} / \mathrm{Fe}_{\mathrm{x}} \mathrm{O}_{\mathrm{y}}{ }^{23}$ systems. Previously, we quantitatively demonstrated the influence of the pre-oxide shell layer on the tensile mechanical properties of pristine Ni NWs. ${ }^{2}$ Our previous study indicates that the intrinsic mechanical properties of metallic Ni NWs subjected to tensile loading strongly depend on the existence of the pre-oxide shell layer and on the size of the NWs. ${ }^{2}$ The softening of the yield strength is related to the pre-oxide shell layer and diameter. ${ }^{2,10,21,23}$ Moreover, we indicated that the pre-oxide shell layer on the free surface of metallic Fe NWs degrades the overall mechanical tensile properties of the pristine NWs associated with the decreasing mechanical stress to initiate plastic deformation as compared to their pristine counterparts. ${ }^{23}$

Since there has been no theoretical and experimental compression study of the pre-oxide shell layer effects on the mechanical deformation properties under compressive loading, the quantitative effect of surface oxidation on the size-dependent properties of pristine Ni NWs remains unknown. In this study, considering the practical importance of pristine and oxide-coated Ni NWs, we carry out MD simulations using the Large-scale Atomic/Molecular Massively Parallel Simulator (LAMMPS) package code to determine the compressive mechanical deformation properties focusing on the size-dependent pre-oxide effect on the intrinsic mechanical deformation properties at each relevant nanoscale. ${ }^{24}$

\section{SIMULATION METHODOLOGY}

We begin by briefly explaining the simulation methodology of oxidation and the subsequent formation of the resulting shell oxide layers on the free surface of pristine Ni NWs. For a detailed description of the oxidation simulations including technical details and initial setup, we refer to our previous report. ${ }^{2}$ To investigate the influence of the pre-oxide shell layer and size-dependent mechanical compressive properties, first, we start with the oxidation simulations by inserting the NWs into a molecular $\mathrm{O}_{2}$ environment to form thin pre-oxide shell layers on the free surface of pristine cylindrical Ni NWs with three different diameters $(D=\sim 5.0,6.5$, and $8.0 \mathrm{~nm})$. All oxidation processes were performed at $300 \mathrm{~K}$ using a canonical ensemble (NVT) with Nose-Hoover thermostat ensemble. ${ }^{25}$ The higher reactive nature of $\mathrm{Ni}$ atoms toward metallic $\mathrm{O}_{2}$ molecules assisted by larger specific surface areas results in a spontaneous formation of the oxide shell layer on the free surface of pristine Ni NWs. During oxidation, the dissociation of $\mathrm{O}-\mathrm{O}$ bonds and segregation, adsorption, and incorporation of $\mathrm{O}$ atoms at the available surface sites in the form of a thin oxide shell layer on the free surfaces of NWs are strongly coupled with the consequence of chemical reaction assisted simultaneous rapid outward and inward diffusion of anions and cations. ${ }^{4,7}$ Finally, oxidation of NWs leads to a spontaneous formation of a unique intrinsic core/ oxide shell of the $\mathrm{Ni} / \mathrm{Ni}_{\mathrm{x}} \mathrm{O}_{\mathrm{y}}$ structure with an $\sim 1.0 \mathrm{~nm}$ thick amorphous oxide shell layer that covers the structure. ${ }^{2}$ The initial diameters of pristine NWs were increased from $5.0,6.5$, and $8.0 \mathrm{~nm}$ to $\sim 6.0, \sim 7.6$, and $\sim 8.9 \mathrm{~nm}$ (see the supplementary material), respectively. ${ }^{2}$ Oxidation at the free surface resulted in the breaking of the symmetry and surface imperfections, and thus, in the highly random distribution of a disordered structure near or at the $\mathrm{Ni}-\mathrm{Ni}_{\mathrm{x}} \mathrm{O}_{\mathrm{y}}$ interface that may assist in the accumulation of dislocation sites as well as increase the probability of initiation of initial dislocations, causing additional softening. ${ }^{2,6}$

We have characterized the short-range local microstructure and chemical changes of the oxide region that is shown to be an amorphous structure. ${ }^{2}$ In addition, our structural analysis reveals that the atomic rearrangements in the oxide region assisted by complex chemical reactions significantly lead to the overall modification of the size, morphology, charges, types of chemical bonding, density, surface morphology, chemical composition, and local atomic microstructures. ${ }^{2}$ Oxidation is an effective method to manipulate the overall free surface structure of pristine Ni NWs. 
More detailed discussions on the analyzing of structure properties can also be found in our previously published paper. ${ }^{2}$ For instance, the expansion of a surface, e.g., the expansion of the NW volume, leads to the generation of oxide defects, imperfections, and chemical and structural heterogeneities at the surface region and/or the interface, which may act as a driving force to activate the initiation of dislocations below the ideal strength to initiate plastic deformation. As a result, our previous study revealed that Ni NWs are oxidized readily with an approximately average limiting thickness of $\sim 1.0 \mathrm{~nm}$ oxide shell layer with different morphologies on each surface of cylindrical NWs (see the supplementary material). Specifically, the surface oxide shell layer played a decisive role on the initiation of initial dislocation processes in complex ways to initiate plastic deformation that was the main reason behind the softening as well as altering the mechanical deformation properties of the pristine Ni NWs. For example, our previous work indicated that the tensile property of Ni NWs strongly depends on the presence of a pre-oxide shell layer, which lowers the yield strength. ${ }^{2}$

Furthermore, we use the same deformation procedure as outlined in our previous report except the applied loading for all NWs that are compressive. Before the compressive deformation, all NWs are relaxed using the conjugate gradient method (energy minimization) and then equilibrated at $300 \mathrm{~K}$ and zero stress in the axial (z) direction using the Nose-Hoover isobaric-isothermal ensemble (NPT) thermostat and a barostat to achieve a stress-free state. ${ }^{25}$ Following equilibration and relaxation, all Ni NWs are separately compressed uniaxially with a constant strain rate of $0.01 \% \mathrm{ps}^{-1}$ $\left(10^{8} \mathrm{~s}^{-1}\right)$ until the total compressive strain reaches $14 \%$. Monotonic external compressive loading is performed under the canonical (NVT) ensemble at $300 \mathrm{~K}$ along the [001] direction of the NWs. ${ }^{26}$ The loading direction is maintained as periodic, while the other two directions are nonperiodic. Throughout the simulation, engineering average stress of the system is obtained using the Virial theorem. $^{27}$ The equations of motion are integrated using the Velocity Verlet method with a time step of $0.5 \mathrm{fs} .^{2}$

\section{RESULTS AND DISCUSSION}

\section{A. Compressive mechanical properties}

First, we focus on the size effects on the average compressive mechanical properties of the cylindrical pristine NWs with three different $D=\sim 5.0, \sim 6.5$, and $\sim 8.0 \mathrm{~nm}$ at room temperature under compressive loading at a constant strain rate of $0.01 \% \mathrm{ps}^{-1}\left(10^{8} \mathrm{~s}^{-1}\right)$. The engineering stress-strain curves in Fig. 1 indicate a strong size dependence, i.e., the bigger diameter is much stronger than the smaller one. We find that the diameter of the NWs is associated with the engineering stress-strain response as well as average maximum yield stress (maximum critical yield stress) and strain (maximum critical yield strain) of the pristine NWs. The decrease in the diameter weakens the NWs, and materials fail at lower yield strains. Note that in experiments, there is compelling evidence to show the trend of "smaller is stronger" in nanosized metals, which seems to be conflicting with the present simulations. The reason is possibly that most of the experiments are in the dimensions where preexisting dislocations plasticity still dominates the deformation, and the strength is accommodated by the slip plasticity. However, our simulations are in an extremely small regime where dislocation

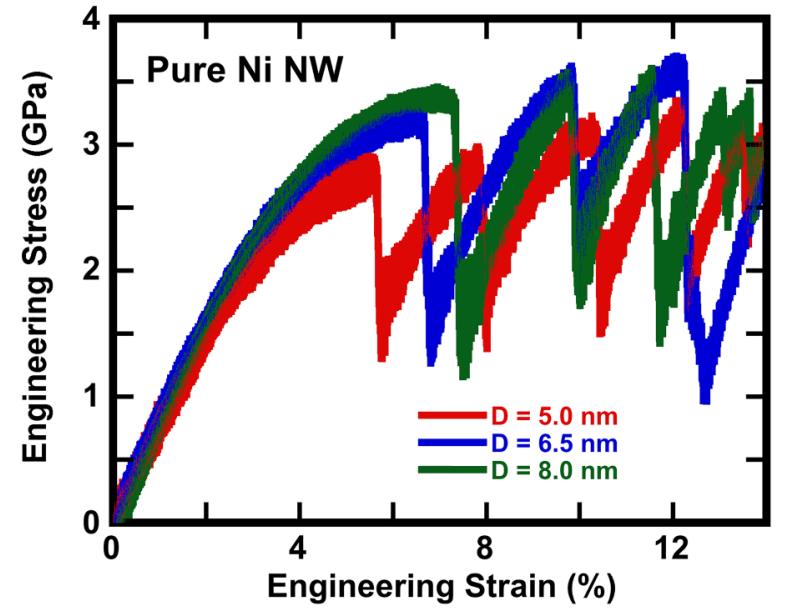

FIG. 1. Characteristic average engineering stress-strain curves for the pristine $\mathrm{Ni}$ NWs of varying diameters. This indicates a clear size effect in the yield stress and strain from the engineering stress-strain curves.

nucleation is the underlying mechanism of the onset of yielding. ${ }^{28}$ In this case, the yielding stress should decrease with decreasing size owing to the appearance of strong surface stress, ${ }^{29,30}$ which promotes the nucleation of dislocations by lowering the activation barrier due to the existence of stress concentration on the surface. In particular, our adopted cylindrical NW models possess a higher quantity of under-coordinated atoms compared to faceted NWs. The under-coordinated atoms could be the favorable sources of dislocation nucleation, which lowers the critical resolved shear stress on the slip planes in the inner core of the NWs.

The engineering stress-strain relationship of all pristine NWs can be characterized into three distinct compressive deformation regions: an initial linear elastic, a nonlinear elastic, and a plastic region. The stress-strain curve is in the predominantly linear elastic region up to in the range of strain $<\sim 3.7 \%-4.1 \%$ (at a stress of $\sim 2.4-2.6 \mathrm{GPa}$ ) and subsequently exhibits nonlinear behavior at larger elastic strains under uniaxial compressive loading. The stress increases with nonlinear behavior to maximum compressive yield stress values of $\sim 2.8, \sim 3.2$, and $\sim 3.4 \mathrm{GPa}$ at a yield strain of $\sim 5.7$, $\sim 6.7$, and $\sim 7.4 \%$ for $\mathrm{D}=\sim 5.0, \sim 6.5$, and $\sim 8.0 \mathrm{~nm}$, respectively. Beyond the maximum compressive yield stress, we observe a sharp drop and subsequent gradual rise again in the stress, which are associated with the nucleation of dislocation and structural rearrangements in the pristine NWs. In the plastic region, the stressstrain curve follows a zigzag (saw-shaped) behavior that can be attributed to the plastic strengthening to accommodate the applied external compressive uniaxial loading with the increasing strain. It can be seen that the stress behaves as oscillating with the zigzag shape even higher than the maximum yielding stress at the initiation of plastic deformation at a higher applied compressive strain.

Next, we analyze the mechanical response of oxide-coated NWs under compressive loading at a constant strain rate. Figure 2 presents the average engineering stress-strain relationships of the oxide-coated NWs as considered in the crystalline core/amorphous oxide shell structured system with $\mathrm{D}=\sim 6.0, \sim 7.6$, and $\sim 8.9 \mathrm{~nm}$. 


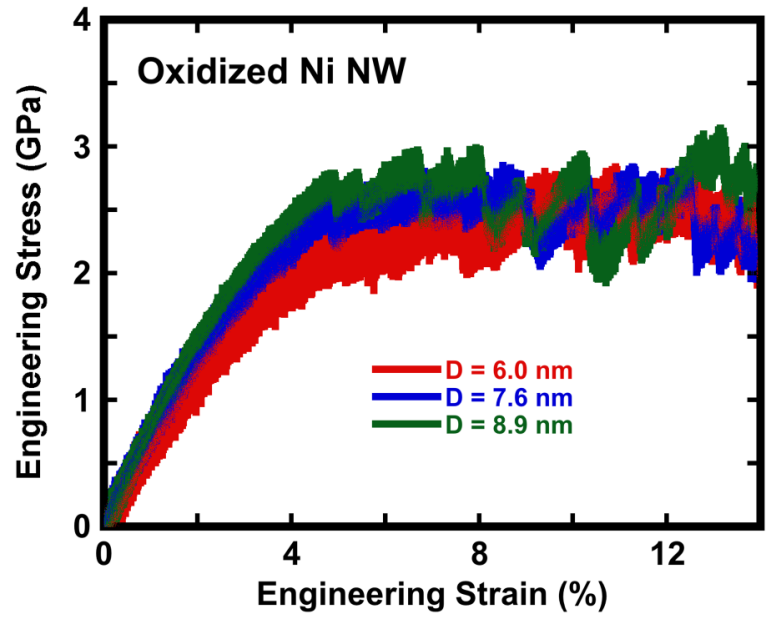

FIG. 2. Characteristic average engineering stress-strain curves for the oxidecoated Ni NWs of varying diameters. This indicates a clear size effect in the yield stress and strain from the engineering stress-strain curves.

Notably, the engineering stress-strain curves exhibit subtle differences. Most importantly, contrary to the pristine NW case, it reveals that the stress-strain is less sensitive to the diameter of the NWs. Particularly, pre-oxide shell layers are less coupled with the diameter to initiate the dislocations; however, they play a significant role in plastic deformation. The values of the average maximum of yield stress and strain are 2.5 and $2.65 \mathrm{GPa}$ and $4.8 \%$ and $4.9 \%$ for $\mathrm{D}=\sim 7.6$ and $\sim 8.9 \mathrm{~nm}$, respectively, which increase slightly with the increasing diameters compared to the pristine NWs. On the other hand, the first drop in stress profile at the initiation of plastic deformation, i.e., the transition to plastic deformation, is not observed obviously for the smallest diameter $(\sim 6.0 \mathrm{~nm})$ from the Fig. 2. Thus, compressive deformation properties associated with the pre-oxide shell layer are unlikely to become important in comparison to the stress-strain curve of the pristine NWs as a function of diameters, since different diameters of the oxide-coated NWs exhibit a relatively minimal difference in the maximum yield stress and strain. The initiation of plastic deformation due to the initiation of initial dislocations becomes relatively easy for the smaller diameter of oxide-coated NWs on corresponding slip planes. The engineering stress-strain curves of oxide-coated NWs also undergo three distinct stages similar to the pristine NWs. The zigzag behavior of the compressive stress-strain profiles accounts for both the diameter and the pre-oxide shell layer, as seen in Fig. 2. However, the zigzag behavior of flow stress is less sensitive to the smaller diameter, while the amplitude of the zigzag behavior gets relatively bigger as the diameter of oxidecoated NWs increases. Thus, the increase in the diameter of the oxidecoated NWs increases the fluctuations in the predicted stress values. This observation implies that the pre-oxide shell layer associated with the diameter controls the shape of the engineering stress-strain curve.

Finally, the engineering stress-strain curve in Figs. 3(a)-3(c) provides quantitative information to compare with the compressive mechanical properties of the pristine and its oxide counterpart, which are at the equivalent size. Notably, the shape of the engineering stress-strain curve for the pristine and its oxidized counterpart is quite different. Here, the oxide-coated pristine Ni NWs exhibit complex dynamics that results typically in a signature associated with a locally evolving highly disordered oxide shell layer having a thickness of $\sim 1 \mathrm{~nm}$, which illustrates that the mechanical deformation behavior is significantly different from its pristine counterpart. In particular, this illustrates that the mechanical compressive properties of the pristine Ni NWs are adversely affected by the presence of the pre-oxide shell layer under the same external uniaxial constant compressive loading condition. The dislocation sources on the oxide layer including the interface apparently operate at stress levels below that associated with the operation of surface sources and/or interfaces. Such a softening can be ascribed to the occupation of the pre-oxide shell layer on the free surface, which facilitates the generation of dislocation sources to the initiation of plastic deformation. Thus, the existence of an oxide shell layer is a dominant factor dictating the strength of Ni NWs, suggesting that the amorphous oxide shell affects the overall mechanical properties and the behavior of the pristine NWs. Furthermore, the initial dislocation sources in the pre-oxidized NWs operate apparently at stress levels below than that of its pristine counterparts. Thus, the pre-oxide shell layer becomes a dominant factor to softening of the pristine Ni NWs. The large initial stress peak indicates the need of
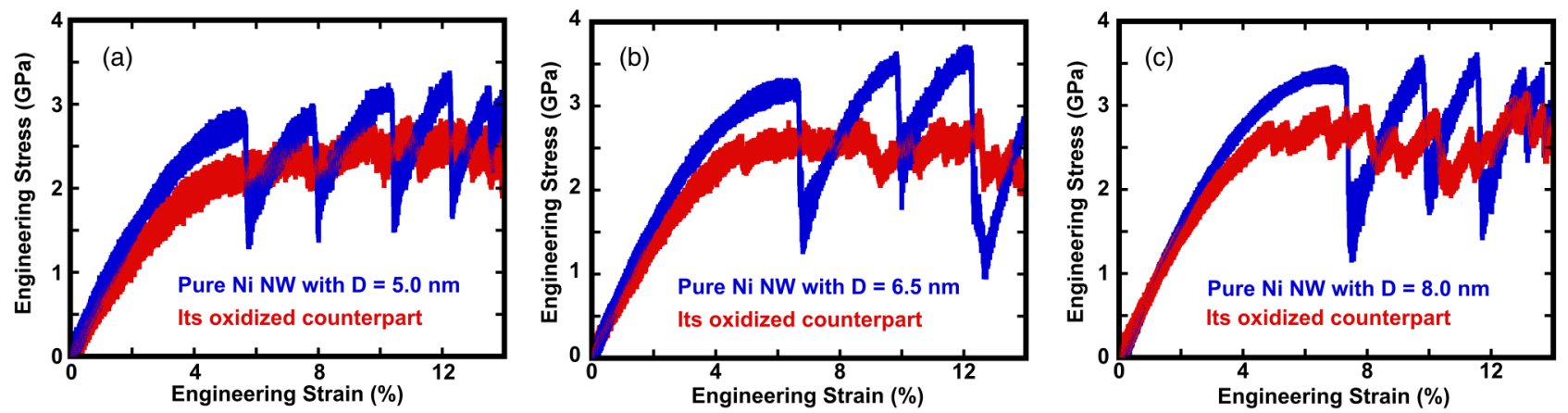

FIG. 3. The quantitative comparison of the stress-strain curve in between the pristine NWs and their pre-oxidized counterparts with the pristine NW diameters: (a) $5.0 \mathrm{~nm}$, (b) $6.5 \mathrm{~nm}$, and (c) $8.0 \mathrm{~nm}$. 
a highly compressive mechanical load to start the plastic deformation. Our results indicate that yield stress and strain values for the oxide-coated NWs decrease as compared to the pristine NWs. Both the pristine and its oxide-coated counterpart retain the unique signature in their stress-strain curves and exhibit three different regimes: linear elastic, nonlinear elastic, and plasticity regime. The stress-strain curves are initially linear with the strain. On the other hand, after the linear regime and before the initiation of plastic deformation, there is a relatively significant change in the slope of the stress-strain curve in both the pristine and its oxide counterpart. All NWs exhibit a nonlinear elastic behavior occurring before the initiation of plastic deformation. After the initiation of plastic deformation, the stress of pristine NWs fluctuates dramatically, but these fluctuations are not significant for the oxide-coated Ni NWs. In comparison with the oxide-coated NWs, the stressstrain curve of the pristine NWs exhibits a considerably larger range of nonlinearity beyond the proportional limit. There is a significant and predominant stress drop to the initiation of plastic deformation and also stress fluctuation in the plastic regime for the pristine Ni NWs. Unlike the pristine NWs, it should be noted that the transition of the deformation mechanisms at the critical compressive stress level is relatively smooth instead of rapid fluctuations, especially for the smaller diameter, as shown in Fig. 3(a). For the oxide-coated NWs with the smallest diameter, Fig. 3(a) shows no sharp, yielding point to the initiation of plastic deformation.

The surface pre-oxide shell layer has a pronounced influence on the mechanical compressive deformation properties. The response of yielding strain to the initiation of plastic deformation of the oxide-coated NWs is much shorter than that of its pristine NW counterparts. The pre-oxide shell layer has a greater effect on the compressive mechanical deformation properties as compared to the stress-strain curve for each pair of pristine and its oxide counterparts. The pristine Ni NWs are more difficult to deform as compared to its oxide-coated counterparts under the uniaxial compressive loading. The pristine NWs exhibit higher maximum yield stress. In addition to that, the average compressive yield stress and strain are highly sensitive in regard to the diameter. For example, the compressive strength of the oxide-coated NWs with $\mathrm{D}=\sim 7.6$ and $\sim 8.9 \mathrm{~nm}$ are $\sim 21.9 \%$ and $\sim 22.0 \%$ lower than its pristine counterparts with $\mathrm{D}=\sim 6.5$ and $\sim 8.0 \mathrm{~nm}$, respectively. More importantly, the preoxidation effect on the yield stress results in roughly $22 \%$ reduction in the compressive strength. It is also revealed that the initiation of initial dislocations for the oxide-coated NWs indeed occurs at the stresses that are well below the maximum yield stress of the pristine Ni NWs. Importantly, the pre-oxide effect on the mechanical properties of NWs is very high, and thus, the surface oxidation effect is not negligible. The incorporation of metallic Ni-amorphous oxide shell layers that consisted of $\mathrm{Ni} / \mathrm{Ni}_{\mathrm{x}} \mathrm{O}_{\mathrm{y}}$ phase boundaries at the interface including the different complex microstructures may create a new intrinsic characteristic to the softening of the pristine Ni NWs, significantly lowering the tensile strength of Ni NWs.

\section{B. Size-dependent mechanical properties analyzed by using common neighbor analysis (CNA)}

Previous theoretical studies indicated that plastic deformation always starts mechanically through emission of initial dislocations from the free surface, oxide shell layer, and/or the interface of the NW for the pristine and oxide-coated NWs that affect the overall mechanical properties. ${ }^{2,23,31}$ However, it is difficult to identify exactly the location of initiation of initial dislocations. Generally, initial dislocation sources activated from these sites to initiate plastic deformation is associated with the combined effects of the geometry, the presence of local defects, the local atomic structure, the thickness of the oxide shell layer, the interface, surface morphologies, the size of the NW, and the applied strain rate. ${ }^{2,21,22,31}$ Therefore, the basic driving force for size-dependent mechanical deformation is believed to arise from the free surface, the oxide region, and/or the interface. Therefore, the low yield strength value of the oxide-coated NWs compared to their pristine counterparts and the softening as a function of diameter can be explained by the local structural analysis at the vicinity of oxide region and the free surface of NWs. The local crystalline structure and defects were determined by using the common neighbor analysis (CNA) in OVITO. $^{32}$ Based on the CNA analysis, the overall percentage of unknown structure atoms are $17.7 \%, 13.3 \%$, and $10.9 \%$ in the vicinity of free surface region for the pristine NWs and $55.6 \%, 44.6 \%$, and $34.9 \%$ in the vicinity of oxide region for the oxide-coated NWs having a thickness of $\sim 1.0 \mathrm{~nm}$ as the diameter increases, as seen in Figs. 4(a)-4(1). In addition, the overall percentage of FCC and unknown (other, undefined) structure atoms compete with each other in controlling the deformation of NWs. It is interesting to note that the overall percentage of the unknown structure increased significantly upon the resulting oxidation because of the amorphous nature of the oxide layer. This result is also consistent with our previous local microstructure analysis, which supports that the oxide region is quite different from its pristine NW counterpart including a highly disordered and inhomogeneous structure. Most importantly, the oxide layer occupies primarily a significant portion of the pristine NWs, and thus the overall percentage of unknown structure atoms is much higher in the oxide-coated NWs, especially with a smaller diameter, where the pre-oxide shell layer effects on the mechanical properties become more pronounced. Physically, the difference in the overall percentage of unknown structure atoms between the two types of free surface is a strong indicator to qualitative changes in the mechanical behavior and the mechanical properties in between the pristine and oxide-coated NWs. It indicates an apparent trend that the overall percentage of unknown structure atoms increases with decreasing diameter, e.g., the surface-to-volume ratio becomes correspondingly large. The pristine and oxide-coated NWs appear to be affected in a similar way by a reduction of the overall percentage of unknown structure atoms as a function of diameter. Correspondingly, the overall fraction of the unknown structure, such as the likelihood of the available number of pre-existing initial potential dislocation sources is in relation with the sample size, increases with decreasing the diameter of NWs. Specifically, the overall percentage of the unknown structure (the total number of uncoordinated and/or amorphous atoms) may regularize the probability of initiation of initial dislocations, which make the NWs strength predominantly as a function of their diameters.

In case of the pristine NWs, the construction of a cylindrical shape and subsequently relaxation is more likely to cause some local defects around the free surface, which in turn alter the atomic 


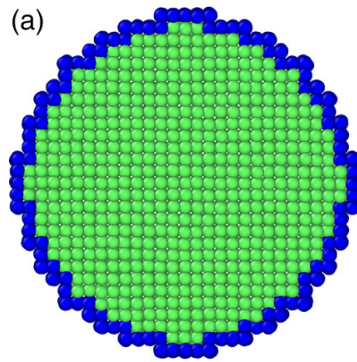

(b)

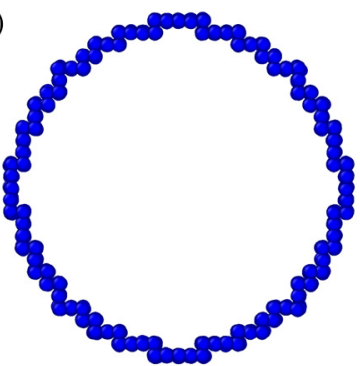

(e)

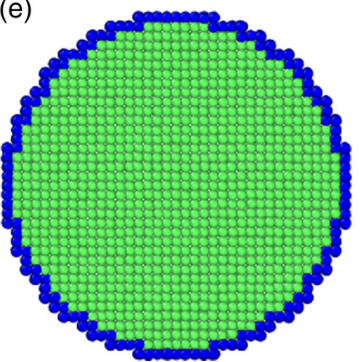

(i)

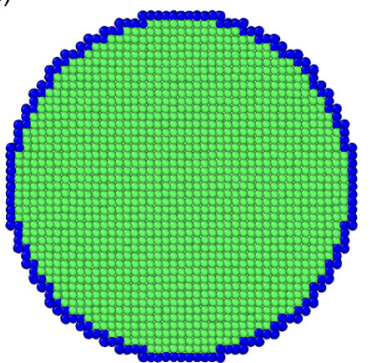

(f)

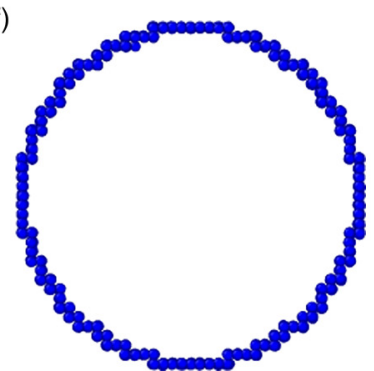

(j)

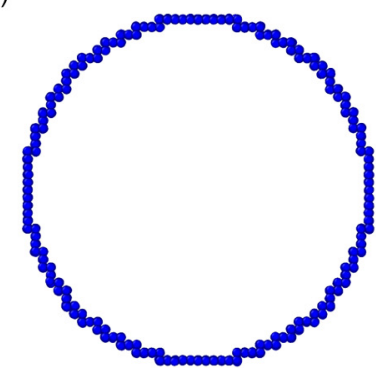

(c)

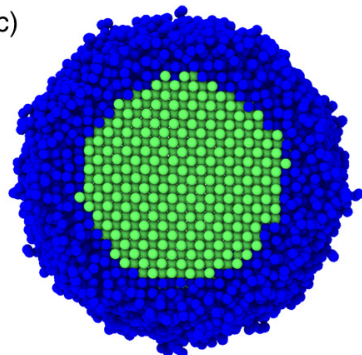

(g)

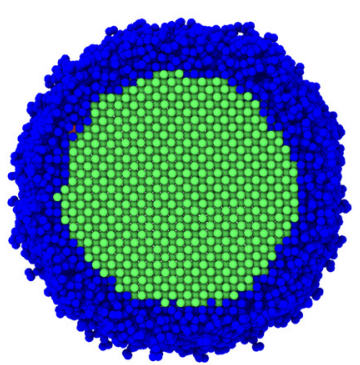

(k)

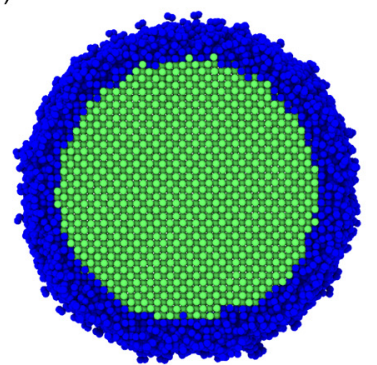

(d)

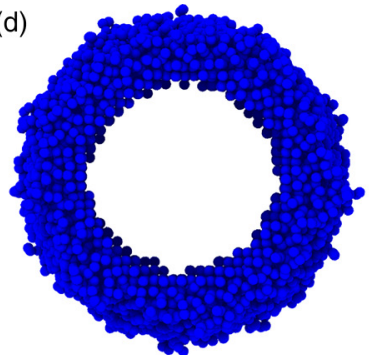

(h)

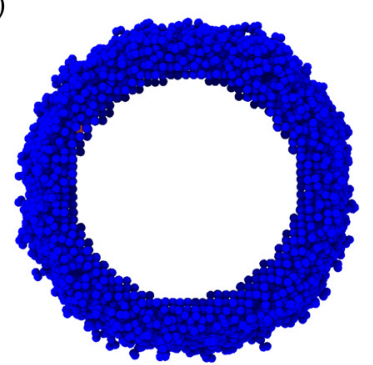

(I)

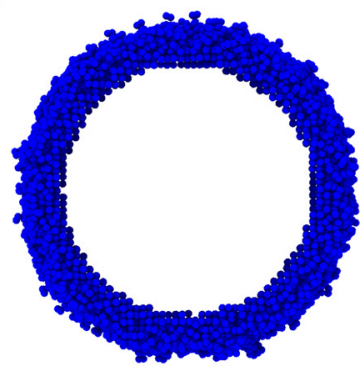

FIG. 4. Cross-sectional views of the local crystalline structure for the [001]-oriented Ni NWs with diameters: (a) $\sim 5.0$, (e) $\sim 6.5$, and (i) $\sim 8.0 \mathrm{~nm}$ and for core-shell $\left(\mathrm{Ni}-\mathrm{Ni}_{\mathrm{x}} \mathrm{O}_{\mathrm{y}}\right) \mathrm{NWs}$ with diameter $(\mathrm{c}) \sim 6.0,(\mathrm{~g}) \sim 7.6$, and $(\mathrm{k}) \sim 8.9 \mathrm{~nm}$, respectively. The local crystalline structure was determined by $\mathrm{CNA}$, where the blue and green spheres represent the unknown and FCC lattice structures, respectively. Based on the CNA analysis, the overall percentage of unknown structure atoms are $17.7 \%, 13.3 \%$, and $10.9 \%$ in the vicinity of the free surface region for the pristine NWs and $55.6 \%, 44.6 \%$, and $34.9 \%$ in the vicinity of oxide region for the oxide-coated NWs as the diameter increases. Snapshots show the unknown structure around the NWs [(b), (f), and (j)] for the pristine NWs as well as [(d), (h), and (I)] for oxide-coated NWs, respectively.

interactions, and thus, could adversely affect the mechanical properties of the pristine NWs. In addition to that, owing to the cylindrical geometric structure of the metallic Ni NWs, the local structure throughout the free surface of NWs is not homogeneous as well. Moreover, other geometric factors such as the morphology of free surfaces are known to affect the initiation of dislocation nucleation. ${ }^{2}$ The unknown atoms can be attributed to the under-coordinated $\mathrm{Ni}$ atoms' structures at the surface associated with the free surface of the pristine NWs. The coexistence of under-coordinated surface atoms (noncrystalline phases) and fully coordinated bulk atoms (crystalline phases) under external compressive loading may be only one of the reasons to incorporate with the emission of the initiation of dislocations to initiate plastic deformation. The relatively high surface-to-volume ratio and associated number of undercoordinated $\mathrm{Ni}$ atoms at the surface has a certain influence on the modification of size-dependent mechanical properties of pristine
Ni NWs. For example, the decreasing diameter of NWs increases apparently the number ratio of under-coordinated surface atoms to the fully coordinated atoms ratio in bulk with increasing surface-to-volume ratio that characterizes the NW properties and indicates the surface effects become significant. Thus, the sizedependent mechanical properties and deformation mechanism of the NWs can be attributed to the available number of undercoordinated surface atoms to fully coordinated bulk atoms. The diameter of the NWs also dictates the quantity of the initial dislocation sites as well as the statistical occurrences of possible initial dislocation sources. In addition, the number of initial dislocation sites decreasing with increasing sample size results in a strengthening effect. Specifically, the initiation of plastic deformation is relatively easier for the smaller diameter-requires a lower amount of stress-than for the bigger diameter, that is, the maximum stress required to activate the possible initial dislocation sources to initiate 
plastic deformation is directly dependent upon the number ratio of under-coordinated-fully coordinated atoms.

In the pre-oxide NW case, the local structure and composition of the free excess surface of the pristine Ni NWs associated with $\mathrm{O}_{2}$ molecules are transformed into amorphous and defective oxide shell layer structures upon oxidation. For the oxide-coated NWs, the unknown atoms can be attributed to the amorphous and defective structures. In addition to the increase of the overall percentage of the unknown structure, the surface defect density and size of the pristine NWs have substantially changed during oxidation. We showed already that the local microstructure and initial dislocation concentration sites in the vicinity of free surface were altered significantly. ${ }^{2}$ Thereby, these unusual features of the pre-oxide shell layer reasonably lead to play a pronounced role on the initiation of initial dislocation activities and consequently on the overall underlying mechanical deformation behavior and related properties of oxidecoated NWs. In addition to that, the resulting oxide-shell layer causes weakening of the bonds at the interface between the metallic core and the oxide shell region. For example, a relatively large number of distinct surface defects and/or local bonds at the interface may generate a large number of initial dislocation nucleation sites around the oxide region that effectively reduce the mechanical resistance to initiate plastic deformation. Notably, the initial dislocations are nucleated from the vicinity of the oxide region and/or interface at stresses below the maximum critical stress value of its pristine counterpart. This effect may also be responsible for assisting the initiation of initial dislocations and subsequently compressive mechanical deformation properties. Free surface and oxide region associated with diameter including a possible change of coordinated/uncoordinated atom number involve a general decrease in the compressive yield stress and strain of the NWs.

The Ni NWs with $\sim 1.0 \mathrm{~nm}$ thick oxide shell layers $\left(\mathrm{Ni}-\mathrm{Ni}_{\mathrm{x}} \mathrm{O}_{\mathrm{y}}\right)$ exhibit distinctly different compressive mechanical response and related properties compared to the stress-strain curve for each pair of pristine and its oxide counterparts as illustrated in Figs. 3(a)-3(c). The existence of the local defect structures, the unreacted-overreacted $\mathrm{Ni}$ atoms, and the overall percentage of unknown structure atoms ratio can explain these unusual differences between the deformation of pristine NWs and its oxide counterparts. Mainly, the intricate interplay between the metallic core with the oxide shell layer region including competition and/or cooperation determines the overall mechanical properties of the oxide-coated NWs. In particular, the size-dependent softening for the oxide-coated NWs associated with the reduction of the free surface of NWs including the higher degree of broken $\mathrm{Ni}-\mathrm{Ni}$ bonds and the symmetry at the oxide region and interface upon oxidation. The formation of the surface oxide shell layer associated with the diffusion of ions results in shrinking the metallic core volume that decreases the number of unreacted $\mathrm{Ni}$ atoms. Particularly, the local interactions between microstructure and defects at the vicinity of oxide region and their number as well as distribution can be naturally accounted for initiation of initial dislocations as well as the subsequent motion of dislocations. The coexistence of crystalline core and noncrystalline oxide phase including the interface is associated with the softening of Ni nanowire under the external compressive force. The softening of the oxide-coated NWs becomes relatively significant to accommodate the imposed compressive mechanical strain by decreasing the diameter and increasing the unreacted-over-reacted $\mathrm{Ni}$ atoms ratio, indicating that the initiation of initial dislocations becomes easier and the surface pre-oxide effects dominate. Specifically, the presence of an oxide shell layer takes control of the initiation of initial dislocation sources, which leads to a transition from strengthening to softening, depending on the diameter of NWs.

\section{Comparison between compressive and tensile mechanical properties}

It is interesting to compare qualitatively the compressive mechanical properties with tensile properties of the pristine and oxide-coated Ni NWs. In both cases, we observed that the initiation of plastic deformation is mechanically initiated by initial dislocations from the free surface, in the vicinity of the oxide region, and/ or interface boundaries of the NWs. However, our results reveal a fundamentally different trend of deformation mechanism and properties under tensile and compressive loading for the pristine and oxide-coated NWs. In contrast to uniaxial tensile deformation, we observed a nonlinear stress-strain curve in the elastic region under the uniaxial compressive deformation. There is a significant difference in between the yield stress and strain under two types of loading modes even in pristine and oxide-coated Ni NWs have the same chemical composition. For example, the previously reported tensile yield stress and strain of the pristine NWs are equal to $10.55,10.50$, and $10.10 \mathrm{GPa}$, and $8.25 \%, 8.45 \%$, and $8.50 \%$ for $\mathrm{D}=\sim 8.0, \sim 6.5$, and $\sim 5.0 \mathrm{~nm}$, respectively. The values of the compressive maximum yield stress values decrease in the range of $\sim 2.8-3.4 \mathrm{GPa}$ at yield strain in the range of $\sim 5.7 \%-7.4 \%$ compared to the tensile case, indicating $\sim 68 \%-73 \%$ reduction of the yield stress compared to the tensile loading. Moreover, the results of compressive loading indicate a dramatic decrease in flow strength compared to the tensile flow strength. In addition to the dissimilarity between the tensile and compressive stress curves, we observe a strong size effect for compression, with the slight distinguishable difference in the yield stresses with the diameter. Similarly, for the oxide-coated NWs, the compressive yield stresses are 2.65 and $2.50 \mathrm{GPa}$, while the tensile values are 7.35 and $6.35 \mathrm{GPa}$ for $\mathrm{D}=\sim 8.9$ and $\sim 7.6 \mathrm{~nm}$, respectively. However, we observe weakening effects in both tension and compression with a decrease in the diameters. In both cases, the yield strength of the oxide-coated NWs has been observed to be much lower than those of its pristine counterparts. The yield strength shows a higher value in compression than in tension for all NWs. The size effects on the overall yield strength in the pristine and oxide-coated Ni NWs strongly depend on the loading modes. Generally, the overall yield strength of all NWs tends to be higher in compression than in tension.

Similarly, Jang et al. reported that the tensile strength of nanocrystalline Ni-W alloys nanopillars is higher than compressive ones by $7 \%-15 \% .{ }^{33}$ Moreover, both compression and tension strengths decrease significantly with decreasing pillar diameter. Rinaldi et al. studied the compressive size effects in the yield behavior of single crystalline metallic Ni pillars. ${ }^{34}$ They obtained the stress-strain curves whose behavior looks similar to our stress-strain behavior: linear and nonlinear plastic behavior in the compressive loading. For NWs having diameters in the range of 130-302 nm, yield stress values are between $\sim 0.8$ and $\sim 2.7 \mathrm{GPa}$. Setoodeh et al. simulated 
the compressive and tensile mechanical properties of FCC Ni NWs with a square cross section in the [100] direction using MD simulations by employing embedded atom potential. ${ }^{35}$ They showed that the compressive yield stress is lower than the tensile yield stress. In addition to that, the stress increases up to a maximum value of $2.75 \mathrm{GPa}$ at a strain of 0.05 and then follows a sharp drop in stress with the zigzag (nonlinear elastic) behavior in the compression loading. Xie et al. calculated the dislocation nucleation stress of single crystal $\mathrm{Ni}$, which is defined as the ratio of the maximum uniaxial compressive/tensile stress (R) required to initiate dislocation. ${ }^{36}$ They showed that the dislocation nucleation stress in uniaxial tension is much higher than that in uniaxial compression along the [100] direction, and the value of $\mathrm{R}$ is 0.5 . However, the dislocation nucleation stress in uniaxial tension is much lower than that in uniaxial compression under loading along the [110] and [111] directions. Heng investigated the size effect on the mechanical behavior of $\mathrm{Au}$ pillars under compression loading at $300 \mathrm{~K}$ by MD simulations with the embedded-atom method potential. ${ }^{37}$ Their results indicate that the yield stress exhibits strong size effects and increases significantly with increasing pillar diameter. Overall, our predicted mechanical properties of the Ni NWs under compressive loading are consistent with the data available in the literature.

\section{Mechanism of plastic deformation}

The details of the two distinct nonlinear elastic and plastic compressive deformation behaviors will be discussed below for the pristine NWs with $\mathrm{D}=6.5 \mathrm{~nm}$ and its oxide counterparts. An obvious nonlinear elastic behavior was observed in the stage of elastic deformation under compressive mechanical loading; see stress-strain curves shown in Fig. 3(b). Actually, further compressive deformation of NWs in the nonlinear region is associated with an apparent softening. The progressive transition and activities from the linear regime to the nonlinear plastic deformation are linked directly to the onset of short-range atomic rearrangements from FCC to BCC and unknown crystal structural transformation (phase transformation). Another reason is possibly the strong surface effect on the NWs, which possess a large surface-to-core ratio. ${ }^{17,38}$ These phenomena are clearly observed throughout the volume of NWs beyond the linear elastic stage of compressive loading in the range of $\sim 4.0 \%$ to $\sim 6.8 \%$ strain level. The progressive transition-linear to nonlinear elastic behavior-proceeds continuously through the atomic disordering in the NWs by the formation of BCC and unknown atoms and ends with the establishment of the initiation of plastic deformation for the pristine NWs. The subsequent change in the lattice local structure throughout the volume of the pristine NWs are randomly occurred with a relatively high applied compressive strain to accommodate the applied strain, wherein strengthening competes with these nonuniform transformations until the formation of stacking faults. The recombination of randomly dispersed BCC structure begins to pile up and becomes more ordered relative to those seen at the vicinity of the free surface of the NWs, which promotes further the activation of partial dislocations. Importantly, ordered BCC structure near the free surface may result in increased stress levels to trigger the initiation of initial dislocations. The initiation of plastic deformation is initiated by partial dislocation emission from the free surface into the core of NWs, accompanied by atomic rearrangements from FCC to unknown, BCC, and HCP transformation on the boundary, as seen in Fig. 5. The total number of BCC structural transformation increases dramatically and progressively as the compressive stress increases till the formation of first stacking fault on the (111) plane, e.g., the initiation of plastic deformation, resulting in relatively sudden drops in stresses. As compressive strain increases, the transformation of BCC structure increases from $0 \%$ to $1.6 \%$ (at a strain of $\sim 6.8 \%$ ) for the pristine NWs with $\mathrm{D}=6.5 \mathrm{~nm}$, which indicates that the transition of the main deformation mechanism from nonlinear elastic to plastic deformation mechanisms. Followed by the transition, the number of HCP atoms increases significantly up to $11.9 \%$, in where the FCC type of atoms is around $75 \%$. At the same time, the number of BCC atoms quickly drops down to zero.

First, partial dislocations with two atomic layers on the (111) slip plane at the opposite surfaces of pristine NWs is nucleated and then glided through the NW, which causes the formation of stacking faults behind them. Subsequently, with further compressive strain, two new partial dislocations with two stacked atomic layers
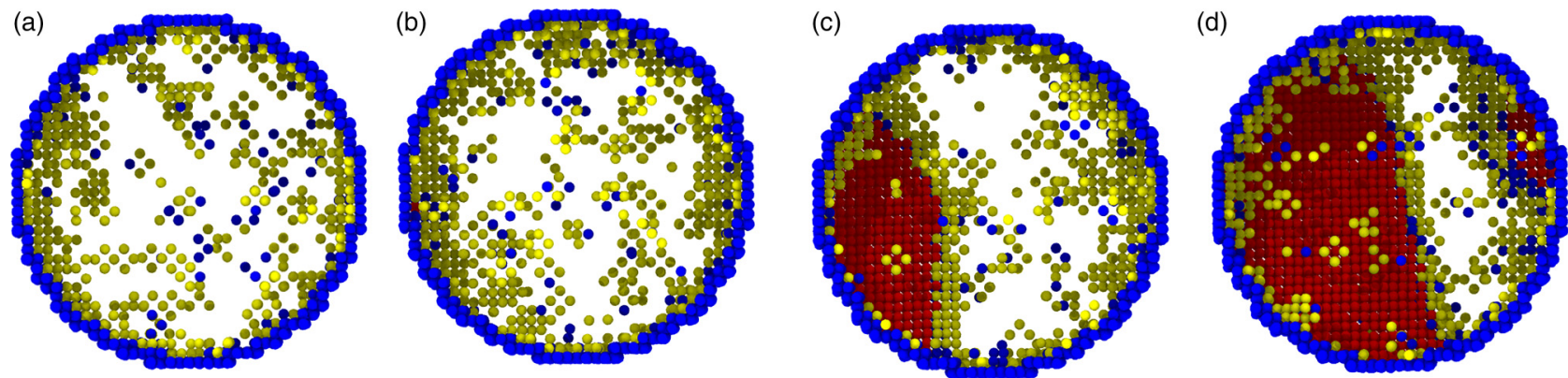

FIG. 5. The reconstruction of the lattice for FCC crystals to absorb the mechanical compressive loading through atomic rearrangement. Atoms at the surface maintain the unknown structure due to cutting the cylindrical shape of the NW and then significant surface reconstruction during relation. Notably, it shows that BCC atoms near the surface maintain most ordered BCC arrangement before the emission of partial dislocations. The increase in the "HPC" type of atoms during the transition from the nonlinear elastic deformation to the plastic regime corresponds to the generation of partial dislocations as well as stacking faults. The unknown, BCC, and HCP structure atoms for the pristine NWs with a core diameter of $6.5 \mathrm{~nm}$ are colored with blue, yellow, and red, respectively; the atoms in FCC crystals are not shown for clarity. 
are emitted at the nearly same time at the opposite ends of the free surface of the NW [Fig. 6(a)]. They propagate rapidly into the NW at the opposite surfaces on the $(-111)$ plane and then formed the stacking faults to generate a twin band, as seen in Fig. 6(b). During this process, increasing compressive strain leads to splitting of two layers dislocation belonging to the first stacking faults into one layer stacking fault to form the twinning. As a result, the compressive stress drops suddenly. Then, it thickens gradually by the smooth migration of twin boundaries, contributing to plastic deformation. Consequently, the creation and propagation of twins coupled with the free surface as well as the oxide region result in the lattice reorientation with respect to the relative
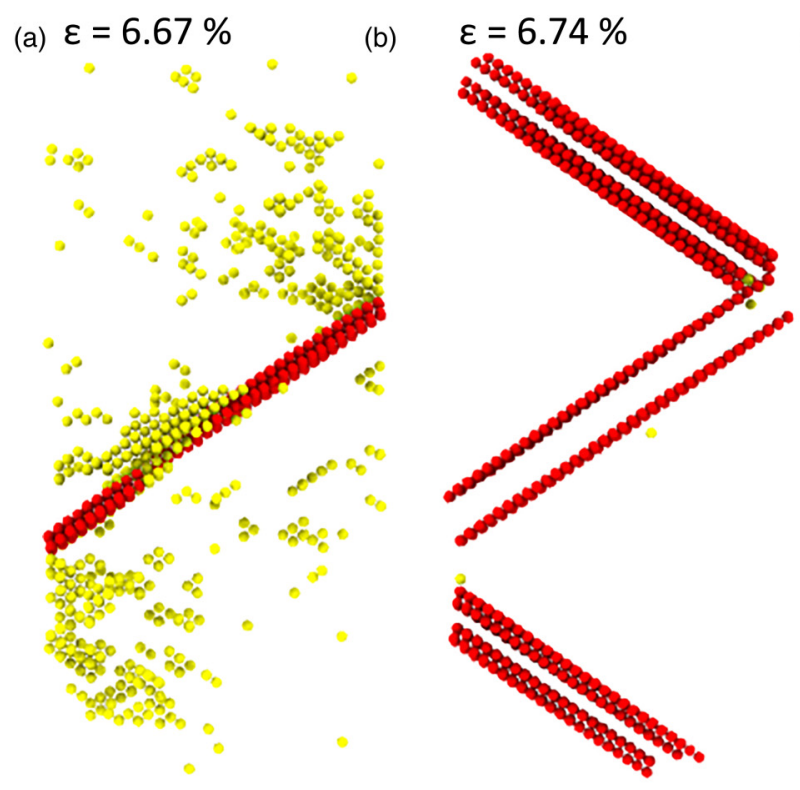

(c)
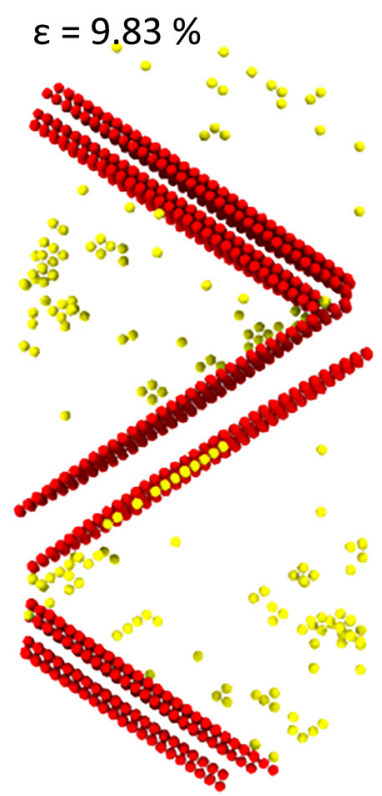

(e)
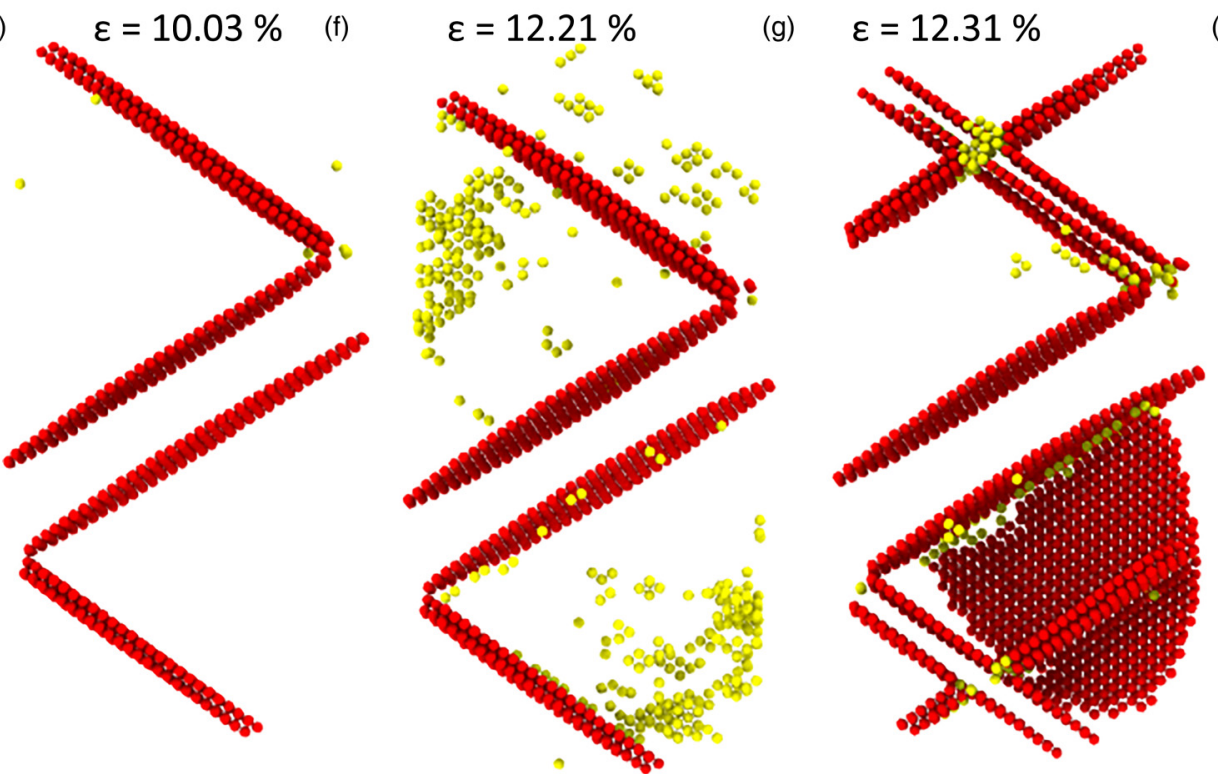

(d)

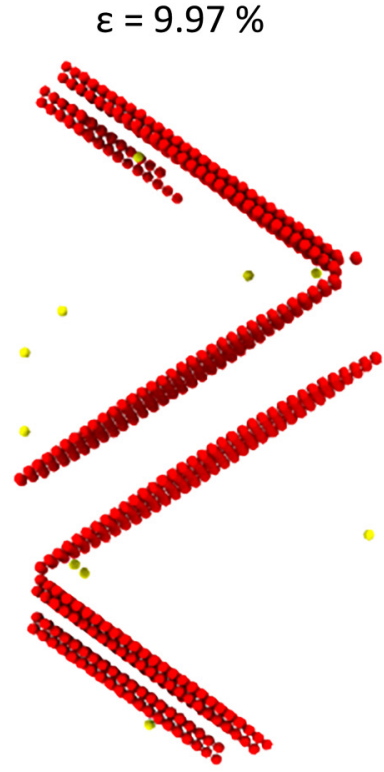

(h)

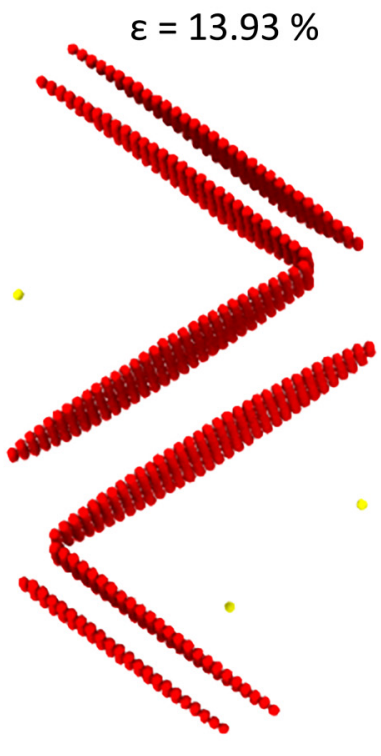

FIG. 6. The evolution of the mechanical compressive plastic deformation at various strain levels during the plastic deformation regime for the pristine NWs with a diameter of $6.5 \mathrm{~nm}$ are colored with red. Unknown and FCC atoms are hidden intentionally. Notably, it shows that BCC atoms through the volume of the NW increase before the deformation activities such as the partial emission dislocation. Significant deformation twinning is observed after the initial surface partial dislocation nucleation. Here, the body-centered cubic (BCC) and hexagonal close-packed (HCP) atoms are colored yellow and red, respectively. 
initial orientation of NWs. Twin configurations associated with stacking fault structures are clean and parallel with each other, as seen in Figs. 6(a) and 6(b). It is noticeable that after the formation of first two stacking faults, the NW was not able to retain the BCC structure and returned into the native FCC arrangements at relatively high strain levels, as seen in Fig. 6(c). However, as displayed in Fig. 6(d), the elimination of one pair of second stacking faults with two atomic layers (one twin boundary) leads consequently to the resulting increase in the stress. As a result, only one of the twin planes survives from the second twin [Figs. 6(a)-6(h)]. Before the transition, transformations of BCC structure are gradually reactivated and, as a consequence, contribute to this elimination and the formation of stacking faults at the much larger applied compressive stress. Similarly, Guenole et al. investigate the role of the silicon (Si) amorphous shell on the process of dislocation nucleation and the plasticity of Si NWs under uniaxial compressive strain using MEAM-Baskes potential. ${ }^{14}$ In core-shell NWs, they observed that the yield strain for the initiation of plasticity, e.g., the nucleation of the first dislocation, which is initiated from interface defects in core-shell NWs, decreased for NWs with an amorphous shell as compared to the pristine NWs. Moreover, the presence of a native crystalline-amorphous interface acts as seeds for the initial dislocation nucleation.

Thus, the formation and elimination of stacking faults are associated partially with zigzag behavior of the stress-strain curve. Furthermore, these transformations of BCC structures were reactivated before emitting new multiple stacking faults at (111) and (11-1), which correspondingly caused the drop in stress. Dislocations traveling across the NW meet with the stacking faults, which encounter obstacles and lead to dislocation splitting. These dislocation-dislocation interactions are associated with the collective formation and/or elimination of stacking faults, contributing to the observed plastic deformation behavior in the NWs. Finally, a less dense population of the well-defined parallel stacking fault planes arrangement is formed and homogeneously distributed within the NWs to accommodate the compressive loading.

The incorporated metallic $\mathrm{Ni}$ with amorphous oxide shell layers, consisting of phase boundaries at the $\mathrm{Ni} / \mathrm{Ni}_{\mathrm{x}} \mathrm{O}_{\mathrm{y}}$ interface including the different complex short-range local atomic structures, creates a new intrinsic characteristic toward the softening of the oxide-coated Ni NWs. Under the external uniaxial mechanical compressive loading beyond the linear elastic regime, the oxidecoated NWs also no longer retain their initial crystalline FCC structure and undergo a BCC structural transformation, which indicates the nonlinear elastic deformation. These transformations for the oxide-coated NWs are activated relatively at the lower applied compressive strain as compared to its pristine counterparts. During the nonlinear elastic region, direct FCC-to-BCC phase transition with further compressive loading randomly occurred and consequently begun piling up close to the interface between the core and the oxide shell layer and/or oxide region, which plays a significant role in mediating initiation of dislocations and effectively lowering the onset of plasticity, shown in Figs. 7(a)-7(c). Intuitively, the interplay between the phase transition and the interface play an additional complex role to initial dislocation sources; the succeeding BCC structural transformation and subsequently partial dislocation are generated in the vicinity of oxide region including the interface between the core and oxide shell layer. Moreover, the BCC and unknown structure transformation assist the partial dislocations movement toward the free surfaces of the NW. Notably, the pre-oxide shell layer region including the interfaces that act as preferential nucleation sites for defects, atomic rearrangement, and local stress concentrations increase the macroscopic compressive load required to initiate plastic deformation. Thereby, a high stress concentration at the boundary between the core and oxide shell layer and/or oxide region triggers initiation of dislocations, which might be a reason why the pristine and oxide counterparts exhibit very different yield behavior to accommodate the applied
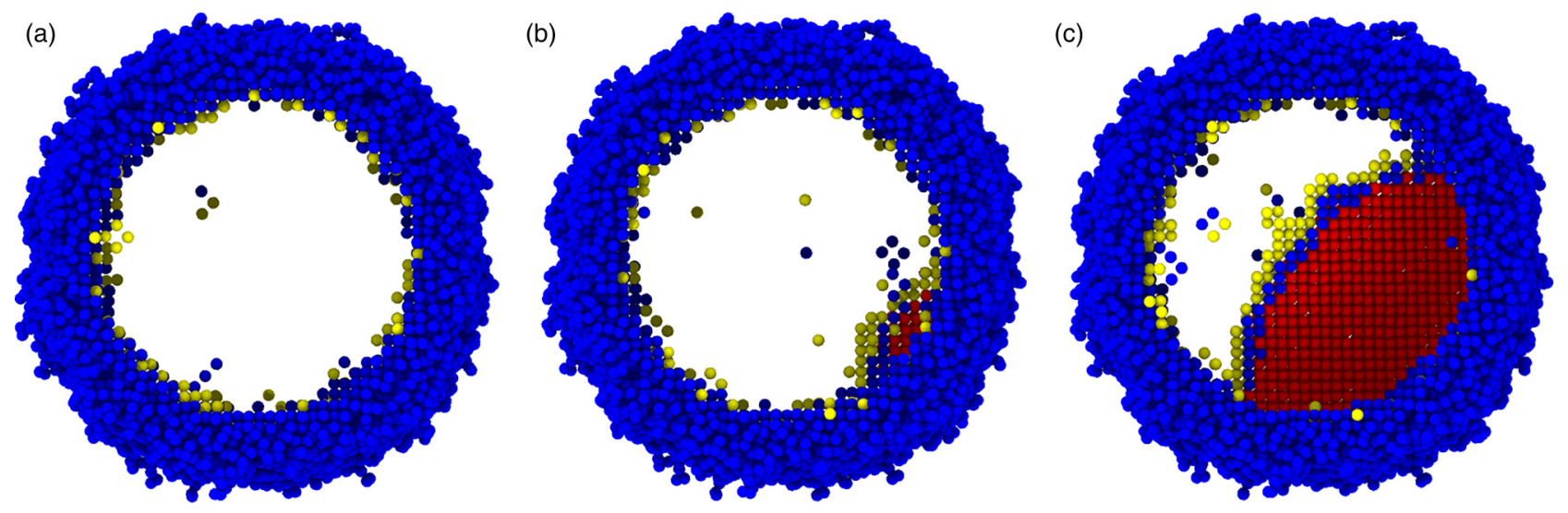

FIG. 7. The distribution of HCP (red), unknown (gray), and BCC (blue) structures in the oxide-coated NWs with a diameter of $\sim 6.7 \mathrm{~nm}$ and a coating thickness of $1.0 \mathrm{~nm}$ at various initial stages of mechanical compressive loading: (a) Just before the initiation of partial dislocation, (b) initiation of initial partial dislocation embryo, and (c) gliding of partial dislocation. Interestingly, the BCC structural transformation mostly occurs at the interface between the metallic core and the oxide shell layer up to the initiation of plastic deformation. Transformation of the BCC structure in the oxide-coated Ni NWs seems to be associated with the interface. The oxide layer results in the destruction of the structural symmetry in the free surface of NW that is allowed to easier rearrangements of BCC structure at the interface. 
(a) $\varepsilon=5.14 \%$

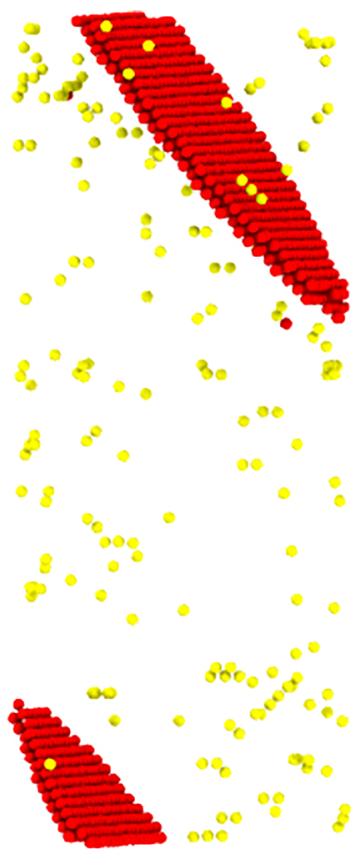

(e) $\varepsilon=6.99 \%$

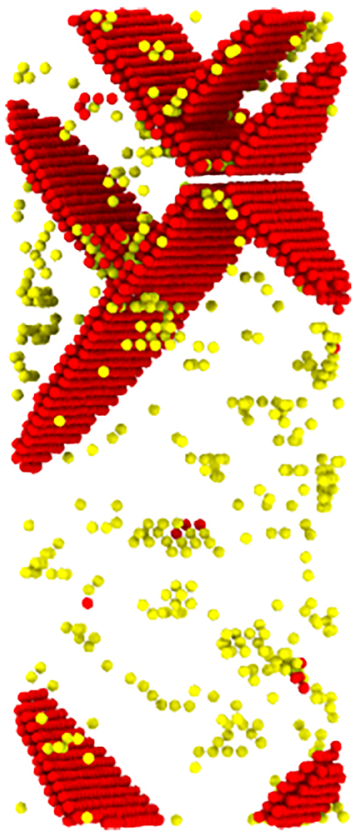

(b) $\varepsilon=5.92 \%$

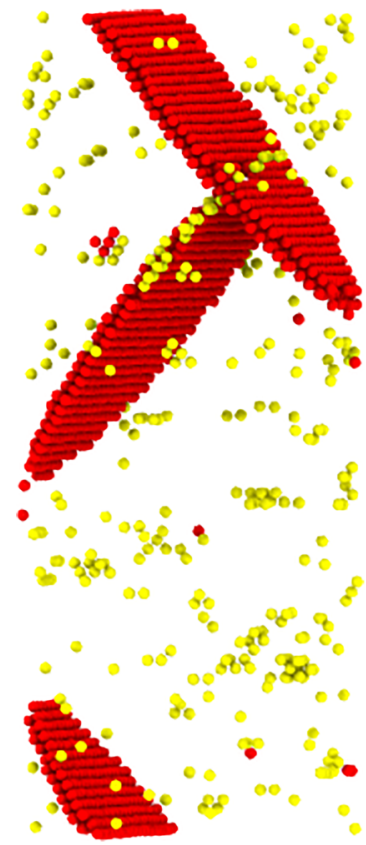

(f) $\varepsilon=7.18 \%$

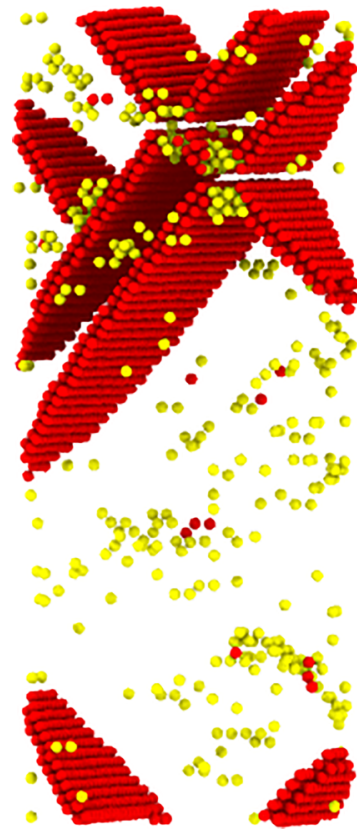

(c)

$\varepsilon=6.62 \%$

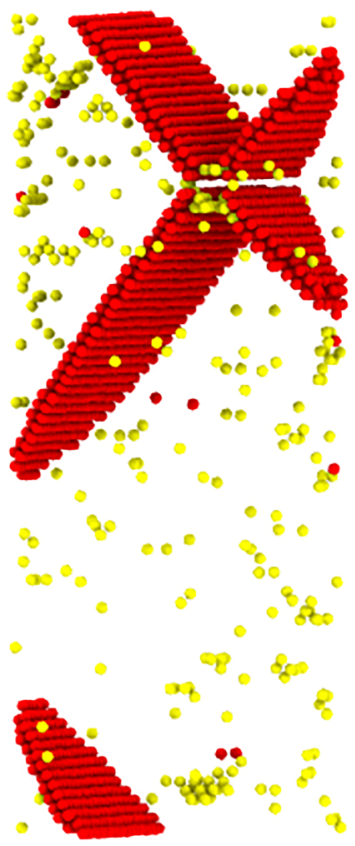

(g) $\varepsilon=7.73 \%$

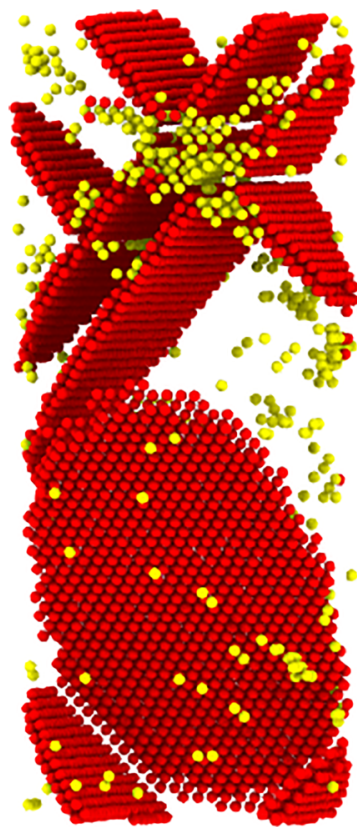

(d) $\varepsilon=6.71 \%$

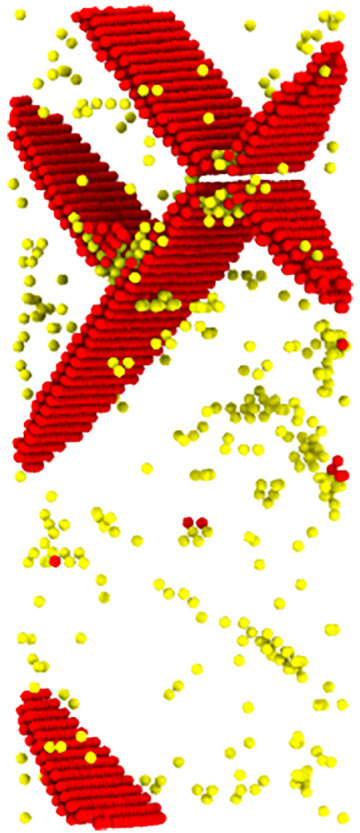

(h)

$$
\varepsilon=13.93 \%
$$

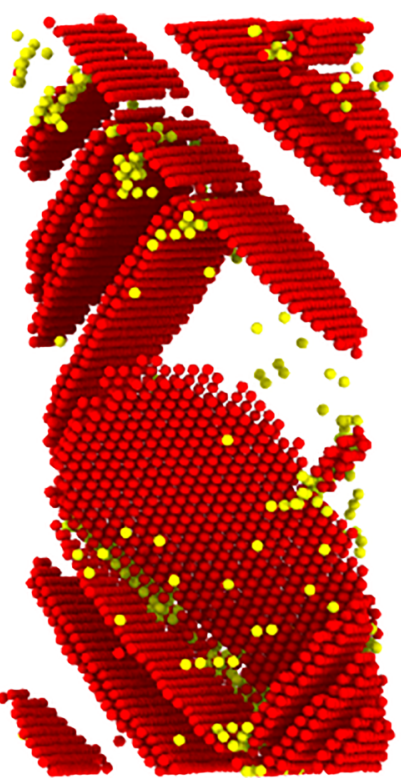

FIG. 8. Evolution details of plastic deformation inside the oxide-coated NWs with $D=6.5 \mathrm{~nm}$ during uniaxial compressive loading at different compressive strain levels The HPC (red) structure transformation indicates the formation and annihilation of partial dislocations and stacking faults. With the increasing compressive loading, the formation of a complex dislocation process repeats by activating the slip systems of the $\{111\}$ slip planes. The pre-existence of the oxide shell layer is attributed to the easier activation of dislocation sources and increased dislocation mobility at the higher compressive strain. The stacking fault structures with spatially distributed stacking fault $\{111\}$ planes appear as perfectly flat interfaces. The presence of a BCC structure during plastic deformation indicates that the deformation mechanism is not the same in the pristine and oxide-coated NWs. 
deformation. We observe that the oxide-coated Ni NWs exhibit different mechanical deformation responses under uniaxial compressive loading, as compared to its pristine counterparts. In particular, beyond the onset of plastic limit, the NW still retains the randomly distributed BCC structure throughout the volume of NWs. This behavior is different from the pristine counterpart, which is typically characterized by compressive softening. The differences in plastic evolution are a result of differences in the initial large defect structures and sites within the oxide-coated NWs in the oxide region.

Deformation starting with the oxide-coated Ni NWs begins with the initiation of partial dislocation with two atomic layers on (1-11) plane at the vicinity of the interface region and quickly grows out to form the stacking faults in this corresponding glide plane [Fig. 8(a)]. During the formation of stacking faults, more and more new BCC structural transformation is activated as the compressive stress increases. In particular, it is noticeable that the oxide-coated NWs are able to retain the BCC structure within the NW interior at relatively high strain levels during all plastic deformations, suggesting the first obvious influence on the NW's plastic deformation mechanisms as well as indicating the difference between the pristine and oxide counterparts of the NW. Moreover, BCC transition and dislocations interact with each other inside the NW during the succeeding deformation. With the increasing compressive strain, the generation of a second partial dislocation from the interface on the $(1-1-1)$ glide plane cuts through the first stacking fault, finally forming a pair of stacking faults. Third partial dislocation nucleation from the free surface glides on the (1-11) plane caused the third stacking faults to intersect with the first stacking faults, which act as obstacles against its dislocation motion under relatively large compressive stress. On the other hand, fourth partial dislocation nucleation from the free surface glides on the (1 -1-1) plane intersected with the first stacking faults, which do not act as barriers to block its propagation through the entire NW. Similarly, new multiple stacking faults are formed on (111) slip planes, as well as the formation of (111) stacking faults within the twins. But it becomes more difficult to interpret their contribution on the plastic deformation mechanism since they interact with each other in a complex way including multiplication of dislocation, elimination, creation, and interaction with the twin boundary that substantially alters the dislocation arrangement by the compressive strain compared to the pristine counterparts. With further increase of the compressive strains, more and more new partial dislocations on the multiple different $\{111\}$ glide planes are similarly activated in a complex way from the interface to form stacking faults inside the oxide-coated NWs, causing dispersed stacking faults with various orientations as seen in Figs. 8(a)-8(h). These partial dislocations and stacking faults on the allowed slip planes eventually interact with the twin boundary during plastic deformation.

The plastic deformation behavior in the oxide-coated NWs is governed by the intense dislocation activity. The dislocation density in the oxide-coated NWs increases continuously with increasing compressive strain throughout the nonlinear elastic and the plastic regions that facilitate dislocation-dislocation interactions. The shorter mixed dislocations segments distributed throughout the pillar volume is predominantly distinct for the oxide-coated NWs. Figures $8(\mathrm{a})-8(\mathrm{~h})$ confirm that the large number of activated slip systems including multiple stacking faults and twins on the $\{111\}$ slip planes seems to be distributed throughout all the volume under applied compressive stress for the oxide-coated NWs. It is suggested that the oxide shell layer has been mediated to increase in dislocation density as well as movement due to dislocation interactions and multiplication.

Similarly, the plasticity of the oxide-coated NWs is associated with the repetitive nucleation, gliding, and annihilation of stacking faults as well as the interactions between dislocations and BCC transformation. Subsequent intermediate collective dislocation events including the formation and elimination of stacking faults as well as the atomic BCC rearrangements are associated with variations in the compressive engineering stress-strain curve with the smaller magnitudes of stress drops and increases (zigzag). In contrast to the pristine counterpart, the zigzag behavior of the stress-strain curve occurs more fluently with the series of small strain for the oxide-coated NWs, which has convincingly been shown to be caused by relatively high collective dislocation activities inside the oxide-coated NWs rather than the dominating twinning mechanism in the pristine $\mathrm{Ni}$ NWs. The latter induces big stress fluctuation that accommodates the pronounced serration in the stress-strain curves of pristine Ni NWs.

Our results mainly indicate that ordinary continuous dislocation activities including creation of stacking faults occur preferably only along the $\{111\}$ favorable restricted slip planes in the FCC crystal of Ni NWs during the compressive plastic regime that is controlled by interactions and reactions of existing dislocations. ${ }^{17,39-42}$ However, eventually different $\{111\}$ slip plane families are activated in order to accommodate the increasing of the applied external compressive strain to carry plastic deformation with the increasing strain. ${ }^{40,42}$ Interestingly, Ni NWs with and without the oxide shell layer display different mechanical deformation characteristics. The differences in compressive mechanical deformation primarily come from the characteristic features of surface oxide. In particular, the oxide shell layer has been attributed to an increase in dislocation density due to dislocation interactions and multiplication. For example, the major difference between the pristine and oxide counterparts of the NW deformation under compression was the number of creation and the randomly dispersed distribution of the stacking fault defects within the NW interior, as seen in Figs. 6(a) $-6(\mathrm{~h})$ and $8(\mathrm{a})-8(\mathrm{~h})$. The pristine NWs show the smaller number of partial dislocations and stacking faults at the end of the simulation. The pre-existence of the oxide shell layer allows the creation of more complex defect structures and the distributed partial dislocation defects and stacking faults, while the deformation by twinning is significantly suppressed as compared to the pristine counterpart.

\section{SUMMARY AND CONCLUDING REMARKS}

In summary, we carried out MD simulations to investigate the effect of pre-oxide shell layers on the compressive mechanical deformation properties of Ni NWs. Our study reveals an interesting correlative relationship between the surface pre-oxide shell layer and the size under compressive loading. We found that the driving force to initiate initial dislocations within the NW is a combination of the applied compressive stress, pre-oxide shell layer, and the size 
of the NW. Weakening trends with the decreasing diameter have been observed for all NWs. Both the diameter and the pre-oxide shell layer play a significant role in the degradation of mechanical properties of Ni NWs. Specifically, the intrinsic compressive strength and properties of the pristine Ni NWs depend highly on the diameter of NWs, such as softening of the NWs observed with the decrease in diameters. The core-shell $\left(\mathrm{Ni} / \mathrm{Ni}_{\mathrm{x}} \mathrm{O}_{\mathrm{y}}\right)$ architecture of the oxide-coated NWs results in lower compressive mechanical properties. Particularly, the pre-oxide shell layer has a significant detrimental influence on the compressive mechanical behavior and related properties as compared to its pristine counterpart. Thus, the deformation can be controlled by a combination of the pre-oxide surface shell layer and the size of NWs. We note that the initiation of initial dislocations to initiate plastic deformation of oxide-coated Ni NWs is associated with the native surface oxide region. In contrast to the uniaxial tension loading, all NWs experience initially linear elastic, nonlinear elastic, and finally plastic deformation regime in the compressive loading.

Consequently, our findings suggest that the incorporation of a pre-oxide shell layer on the pristine NW surface layer caused a degradation of the mechanical properties of the pristine Ni NWs under uniaxial compression. We observed that the characteristics of the surface oxide layers dictate the mechanical properties of the NWs, and thus, require special attention for the utilization of Ni NWs in practical applications. Overall, our results on the prediction of oxidized Ni NWs are a significant step forward toward understanding the oxidation-induced reduction in the mechanical properties of the NWs. The use of reactive potentials to predict mechanical properties at the nanoscale effectively complements the experiments, where the determination of nanoscale mechanical properties is still tremendously challenging.

\section{SUPPLEMENTARY MATERIAL}

Figure 1 in the supplementary material shows the number density and the charge distribution profile for oxygen and nickel atoms along the radial direction, calculated with bins of size $0.5 \AA$.

\section{ACKNOWLEDGMENTS}

This work was supported by The Scientific and Technological Research Council of Turkey (TUBITAK)-BİDEB 2219 through Grant No. 1059B191400364. Simulations were performed at TUBITAK ULAKBIM, High Performance and Grid Computing Center (TR-Grid e-Infrastructure), and ITU National Center for High Performance Computing (UHEM). S.O. acknowledges the support by the Elements Strategy Initiative for Structural Materials (ESISM) and JSPS KAKENHI (Grant Nos. JP18H05453, JP25630013, JP17H01238, and JP17K18827).

\section{REFERENCES}

${ }^{1}$ S. Schrittwieser, D. Reichinger, and J. Schotter, Materials 11, 45 (2017).

${ }^{2}$ G. Aral, M. M. Islam, and A. C. T. van Duin, Phys. Chem. Chem. Phys. 20, 284 (2018).

${ }^{3}$ L. Zhou, A. Rai, N. Piekiel, X. Ma, and M. R. Zachariah, J. Phys. Chem. C 112, 16209 (2008).
${ }^{4}$ S. Garruchet, O. Politano, P. Arnoux, and V. Vignal, Appl. Surf. Sci. 256, 5968 (2010).

${ }^{\mathbf{5}}$ C. Rinsha, C. N. Anumol, M. Chithra, B. N. Sahu, and S. C. Sahoo, AIP Conf. Proc. 1665, 050119 (2015).

${ }^{6}$ L. Barrientos, S. Rodriguez-Llamazares, J. Merchani, P. Jara, N. Yutronic, and V. Lavayen, J. Chil. Chem. Soc. 54, 391 (2009).

${ }^{7}$ N. Amiri and H. Behnejad, J. Chem. Phys. 144, 144705 (2016).

${ }^{8}$ S. K. R. S. Sankaranarayanan and S. Ramanathan, J. Phys. Chem. C 114, 6631 (2010).

${ }^{\mathbf{9}}$ K. Sakiyama, K. Koga, T. Seto, M. Hirasawa, and T. Orii, J. Phys. Chem. B 108, 523 (2004).

${ }^{10}$ M. Tatat, P. Gadaud, P. O. Renault, J. Balmain, C. Coupeau, and X. Milhet, Mater. Sci. Eng. A 571, 92-94 (2013).

${ }^{11}$ B. J. Henz, T. Hawa, and M. Zachariah, J. Appl. Phys. 105, 124310 (2009).

${ }^{12}$ C. Peng, Y. Ganesan, Y. Lu, and J. Lou, J. Appl. Phys, 111, 063524 (2012).

${ }^{13}$ Y. Lu, C. Peng, Y. Ganesan, J. Y. Huang, and J. Lou, Nanotechnology 22, 355702 (2011).

${ }^{14}$ J. Guenole, J. Godet, and S. Brochard, Phys. Rev. B 87, 045201 (2013).

${ }^{15}$ D. Liu, D. Li, and D. Yang, AIP Adv. 7, 015028 (2017).

${ }^{16}$ H. Ikeda, Y. Qi, T. Cagin, K. Samwer, W. L. Johnson, and W. A. Goddard III, Phys. Rev. Lett. 82, 2900 (1999).

17Y. H. Wen, Z. Z. Zhu, and R. Z. Zhu, Comput. Mater. Sci. 41, 553 (2008).

${ }^{18}$ P. S. Branicio and J. P. Rino, Phys. Rev. B 62, 16950 (2000).

${ }^{19}$ S. F. Ferdous and A. Adnan, Comput. Mater. Sci. 90, 221 (2014).

${ }^{20}$ C. Zou, Y. K. Shin, A. C. T. van Duin, H. Fang, and Z. K. Liu, Acta Mater. 83, 102 (2015).

${ }^{21}$ F. G. Sen, Y. Qi, A. C. T. van Duin, and A. T. Alpas, Appl. Phys. Lett. 102, 051912 (2013).

${ }^{22}$ F. G. Sen, A. T. Alpas, A. C. T. van Duin, and Y. Qi, Nat. Commun. 5, 3959 (2014).

${ }^{23}$ G. Aral, Y. J. Wang, S. Ogata, and A. C. T. van Duin, J. Appl. Phys. 120, 135104 (2016).

${ }^{24}$ S. Plimpton, J. Comput. Phys. 1, 117 (1995).

${ }^{25}$ G. J. Martyna, D. J. Tobias, and M. L. Klein, J. Chem. Phys. 101, 4177 (1994).

${ }^{\mathbf{2 6}}$ G. J. Martyna, M. L. Klein, and M. Tuckerman, J. Chem. Phys. 97, 2635 (1992).

${ }^{27}$ M. P. Allen and L. J. Tildesley, Computer Simulation of Liquids (Oxford University Press, New York, 1987).

${ }^{28}$ T. Zhu and J. Li, Prog. Mater. Sci. 55, 710 (2010).

${ }^{29}$ Q. J. Li and E. Ma, Mater. Res. Lett. 6, 283 (2018).

${ }^{30}$ Q. J. Li, B. Xu, S. Hara, J. Li, and E. Ma, Acta Mater. 145, 19 (2018).

${ }^{31}$ G. Aral, M. M. Islam, Y. J. Wang, S. Ogata, and A. C. T. van Duin, Phys. Chem. Chem. Phys. 20, 17289 (2018).

${ }^{32}$ A. Stukowski, Modell. Simul. Mater. Sci. Eng. 18, 015012 (2010).

${ }^{33}$ D. Jang and J. R. Greer, Scr. Mater. 64, 77 (2011).

${ }^{34}$ A. Rinaldi, P. Peralta, C. Friesen, and K. Sieradzki, Acta Mater. 56, 511 (2008).

${ }^{35}$ A. R. Setoodeh, H. Attariani, and M. Khosrownejad, Comput. Mater. Sci. 44, 378 (2008).

${ }^{36}$ H. Xie, T. Yu, and F. Yin, Mater. Sci. Eng. A 604, 142-147 (2014).

${ }^{37}$ T. Q. Heng, Sci. China Phys. Mech. Astron. 55, 1111 (2012).

${ }^{38}$ Y. J. Wang, J. Gao, and S. Ogata, Appl. Phys. Lett. 102, 41902 (2013).

${ }^{39}$ W. Liang and M. Zhou, Phys. Rev. B 73, 115409 (2006).

${ }^{40}$ A. S. Schneider, D. Kiener, C. M. Yakacki, H. J. Maier, P. A. Gruber, N. Tamura, M. Kunz, A. M. Minor, and C. P. Frick, Mater. Sci. Eng. A 559, 147 (2013).

${ }^{41}$ C. P. Frick, B. G. Clark, S. Orso, A. S. Schneider, and E. Arzt, Mater. Sci. Eng. A 489, 319 (2008).

${ }^{\mathbf{4 2}}$ H. S. Park, K. Gall, and J. A. Zimmerman, J. Mech. Phys. Solids 54, 1862 (2006). 